La "era progresista” en Uruguay: ¿cambios o continuidades en la política exterior hacia Brasil?

Resumen: El $1^{\circ}$ de Marzo de 2005, la coalición de partidos políticos progresistas Frente Amplio asumió el Poder Ejecutivo luego de haber ganado las elecciones presidenciales de 2004, acontecimiento electoral y político sin precedentes en Uruguay. La conformación del primer gobierno de izquierda en este país supuso cambios en su política exterior y un nuevo modelo de inserción internacional. A continuación, analizaremos en primer lugar la política exterior uruguaya durante los gobiernos progresistas a partir de una caracterización general de esta y de la dimensión internacional en el programa de gobierno frenteamplista. Seguiremos con una breve reseña de la política exterior de José Mujica y de su relacionamiento con el Brasil de Dilma Rousseff, para finalizar, en un tercer apartado, con la dimensión global, regional y bilateral de las actuales agendas de cooperación.

Palabras clave: Uruguay; Brasil; política exterior; medios de comunicación; OPEU.

The "progressive era" in Uruguay: changes or continuities in foreign policy towards Brazil?

Abstract: In the first day of March 2005, the coalition of the progressive political parties, Frente Amplio, rose to the Executive Power after winning the presidential elections of 2004. Such political and electoral event had no precedent in Uruguay. The consolidation of the first leftist government in the country represented changes in its foreign policy and a new model of international insertion. In the following pages, we analyse Uruguayan foreign policy during the progressive governments from a general characterization of the policy adopted and from the international dimension presented in the frenteamplista government's plan. Afterwards, we follow with a brief analysis of the foreign policy of José Mujica's government and its relationship with Dilma Rousseff's Brazil as well as with in the global, regional and bilateral dimensions of the current cooperation agendas.

Key Words: Uruguay; Brazil; Foreign Policy; Media; OPEU

Artigo recebido em 01/04/2016 e aprovado em 07/04/16.

La dimensión internacional en el programa de gobierno del Frente Amplio y la política exterior de Tabaré Vázquez 


\section{LA “ERA PROGRESISTA” EN URUGUAY: ¿CAMBIOS O CONTINUIDADES EN LA POLÍTICA EXTERIOR HACIA BRASIL?}

RAFAEL ALVARIZA ALLENDE

El $1^{\circ}$ de Marzo de 2005, la coalición de partidos políticos progresistas Frente Amplio asumió el Poder Ejecutivo luego de haber ganado las elecciones presidenciales de 2004, acontecimiento electoral y político sin precedentes en Uruguay. Representada bajo el lema "Frente Amplio-Encuentro Progresista-Nueva Mayoría" y con el 50,3\% de los votos de la ciudadanía, la victoria de la izquierda determinó que Tabaré Vázquez asumiera la Presidencia del país con el respaldo de una mayoría parlamentaria integrada por 17 de los 31 Senadores y de 52 de los 99 representantes (o diputados) de la República.

La conformación del primer gobierno de izquierda en Uruguay supuso cambios en su política exterior y un nuevo modelo de inserción internacional. Esta política exterior, fue una de las tantas políticas públicas que experimentaron cambios frente a las tradicionalmente desarrollas por el Partido Colorado y el Partido Nacional, partidos políticos estos que han gobernado la República durante la mayor parte de su historia.

\section{Artigo recebido em 01/04/2016 e aprovado em 07/04/2016}

Hasta el advenimiento de la llamada "era progresista" (Garcé; Yaffé, 2005) la política exterior uruguaya podría caracterizarse como pacifista, juridicista, pronorteamericana, panamericanista, proisraelí, y proclive a la integración regional (Pérez Antón, 2010). De esta manera, se afirma también que esta política exterior ha cumplido con los principios rectores de defensa de la soberanía de los Estados; de no intervención; de solución pacífica de controversias; de defensa de los derechos humanos; de rechazo al terrorismo, a la violencia y a la discriminación; de cumplimiento de buena fe de los Tratados; de solidaridad internacional; y de multilateralismo que prioriza el sistema de Naciones Unidas como único medio de restablecer la vigencia del Derecho Internacional, de la paz y del fortalecimiento del sistema internacional ${ }^{\mathrm{II}}$.

Sin embargo, con la llegada al poder del Frente Amplio se percibe un cambio en la política exterior del país. En este sentido, un breve análisis del programa de gobierno del Frente Amplio y de las variaciones adaptativas de su contenido de acuerdo al escenario internacional imperante resulta un insumo para comprender este cambio.

La dimensión internacional en los programas de los partidos políticos de izquierda uruguayos tuvo su origen en el internacionalismo de corte marxista; movimiento político con fuertes conexiones con las izquierdas europeas y presente en la base programática de dos de los partidos fundadores del Frente Amplio: el Partido Comunista y el Partido Socialista uruguayo. A su vez, el antiimperialismo, la Teología de la Liberación, el estudio del indigenismo y los movimientos agraristas, la subordinación de América Latina y la Revolución Cubana incidieron fuertemente en la identidad política de la izquierda en el país (Clemente, 2004). A partir de la fundación de la coalición de partidos políticos Frente Amplio en 1971, la izquierda uruguaya comienza a incorporar nuevos temas y problemas propios al contexto regional y al sistema internacional. A partir del estudio de diversos documentos elaborados por el Frente Amplio, Isabel Clemente (Clemente, 2004) identifica claras 


\section{LA “ERA PROGRESISTA” EN URUGUAY: ¿CAMBIOS O CONTINUIDADES EN LA POLÍTICA EXTERIOR HACIA BRASIL?}

RAFAEL ALVARIZA ALLENDE

variaciones en el programa político de la coalición y que inciden directamente en la construcción de su política exterior.

A grandes rasgos, podremos subrayar que, para su fundación, está latente la necesidad de la construcción de un régimen nuevo parte de la lucha por la liberalización y desarrollo del Tercer Mundo y de América Latina en particular ("Declaración Constitutiva y Compromiso Político", 05/02/1971). Luego, durante la transición de la dictadura a la democracia, el Frente Amplio abogaba por una política exterior independiente, por el mantenimiento de relaciones con todos los países, por la reestructura de Cancillería y por un nuevo concepto de integración latinoamericana ("Bases Programáticas", 1984).

En los años siguientes, el principio de solidaridad activa con todos los pueblos que luchan contra el colonialismo y el rechazo a la Organización de Estados Americanos (OEA), a la Asociación Latinoamericana de Integración (ALADI) y al Fondo Monetario Internacional (FMI) como instrumentos del imperialismo y responsables de la dependencia regional, desaparecen como líneas programáticas. Ya en 1991, el Documento de los "24" presenta a la integración de América Latina - y en especial el Cono Sur - como "una respuesta válida y necesaria para enfrentar los nuevos desafíos del sistema internacional en transición"; también allí, surge la idea de consolidar las relaciones exteriores como una política de Estado.

Una década después, el Frente Amplio incorporó nuevas categorías analíticas para la fundamentación de su política exterior. En este sentido, se destaca la problemática de la globalización - concibiéndola como el resultado de la revolución científica y tecnológica que determina el desarrollo de las comunicaciones y la profundización de asimetrías entre dominados y dominadores - y se examina al neoliberalismo como una ideología que se presenta como inexorable y negadora de un pensamiento alternativo. Para ello, se proponen tres respuestas, a saber: la articulación de los movimientos progresistas del mundo; la democratización del conocimiento y de los medios de comunicación; y la promoción de la integración regional como estrategia para interactuar con los principales bloques económicos. Aquí, nace la necesidad de reconfigurar el MERCOSUR como instrumento de los movimientos progresistas y de los intereses nacionales ("Pautas para el desarrollo ideológico y la elaboración programática, 2001).

En este sentido plantea de Clemente (2004):

\footnotetext{
Esta revisión de textos revela la persistencia de los temas y supuestos principales en el proyecto de política exterior de la izquierda desde 1971 a 2004 y permite establecer que los elementos de cambio están directamente condicionados por los factores externos que los propios documentos constatan parcialmente, en particular los que tienen que ver con el cambio en el orden internacional: el derrumbe del campo socialista, la desarticulación del Tercer Mundo y del movimiento de No Alineados, la restauración democrática en América Latina, la conversión de la mayoría de los movimientos insurgentes latinoamericanos en partidos políticos, la creación de nuevos espacios para el ejercicio de una política exterior autónoma de América Latina con la iniciativa de Contadora y el Grupo de Río, la emergencia de nuevas economías capitalistas en el Sudeste Asiático y la vertiginosa transformación de China e India en grandes potencias económicas ${ }^{\text {III }}$.
} 


\title{
LA “ERA PROGRESISTA” EN URUGUAY: ¿CAMBIOS O CONTINUIDADES EN LA POLÍTICA EXTERIOR HACIA BRASIL?
}

\author{
RAFAEL ALVARIZA ALLENDE
}

Para las elecciones nacionales de 2004, el Frente Amplio presentó como programa de gobierno una "Recopilación de las propuestas, proyectos e ideas para el gobierno progresista formuladas por el Dr. Tabaré Vázquez en el ciclo 'la transición responsable'", destinando al capítulo "Uruguay Integrado. En el MERCOSUR y en el mundo" sus principales lineamientos en materia de política exterior . IV

El programa de gobierno del Frente Amplio - que tenía a Tabaré Vázquez como candidato presidenciable - se inicia resaltando los principios y valores que hacen a la política exterior uruguaya, para continuar con su proyecto de inserción en el sistema internacional y el desarrollo de una política de comercio exterior, seguido de la importancia del MERCOSUR y de los procesos de integración regional, para finalizar con la necesidad de reforma del Ministerio de Relaciones Exteriores y otros temas de política exterior donde se destaca la defensa y promoción activa de los derechos humanos.

El documento señala que los vínculos históricos, la estabilidad política y el desarrollo económico, así como la circulación de personas, determinan que la primera relación con el exterior sea con Argentina y Brasil, países "hermanos y vecinos", con quienes buscará fortalecer las relaciones bilaterales y la integración como un todo, sin contradecir una política de inserción internacional activa. Además de pilar para la integración regional, el MERCOSUR aparece como un bloque necesario para negociar frente al Área de Libre Comercio de las Américas (ALCA), a la Unión Europea y a otros ámbitos multilaterales. Pero también la OEA, las Cumbres Iberoamericanas y la cooperación sur-sur son destacados como instrumentos para la inserción internacional.

La victoria de Tabaré Vázquez y su asunción el $1^{\circ}$ de Marzo de 2005 implicó el desarrollo de una política exterior de pragmatismo divida entre el relanzamiento del MERCOSUR bajo el lema "más y mejor MERCOSUR" y la búsqueda de nuevos socios estratégicos como fue el caso de Estados Unidos. Su agenda exterior estuvo, en cierta medida, dominada por el conflicto con Argentina por la instalación de plantas procesadoras de celulosa en la frontera, así como también por la negociación de un Tratado de Libre Comercio con Estados Unidos en 2006.

Para Carlos Luján, la agenda internacional del primer gobierno progresista estuvo marcada por tres temas que construyeron el debate acerca de la política exterior del país. En primer lugar, ubica las relaciones con Argentina, Brasil y Estados Unidos, en segundo, la definición del espacio regional - MERCOSUR, Sudamérica o Latinoamérica - prioritario para el país, y en tercer lugar, se refiere a los bloques económicos y políticos por los cuales el país debía inclinarse: Asia, Europa o Norteamérica (Luján en Quiroga; França, 2010:136-176).

Con respecto al primer tema, cabe destacar que el diferendo con Argentina consumió importantes energías de la diplomacia uruguaya durante el gobierno de Vázquez. Alcanzando el punto más álgido en 2006, implicó la demanda argentina ante la Corte Internacional de Justicia de La Haya, donde se acusaba al país de violar el Estatuto del Río Uruguay al autorizar la instalación plantas procesadoras celulosa en Fray Bentos. El pedido de Argentina de reubicación de plantas ante la OEA y el Banco Mundial y posteriormente la solicitud de suspensión de estas obras, así como el bloqueo de dos puentes binacionales por ambientalistas 


\section{LA “ERA PROGRESISTA” EN URUGUAY: ¿CAMBIOS O CONTINUIDADES EN LA POLÍTICA EXTERIOR HACIA BRASIL?}

RAFAEL ALVARIZA ALLENDE

que causaron importantes pérdidas a la economía uruguaya, así como la creación de un Tribunal Ad hoc en el MERCOSUR para tratar el tema - y su pronunciamiento ambiguo e incapaz de resolver el conflicto - se tradujo en el desarrollo de una tesitura de firmeza y reafirmación soberanista del gobierno frenteamplista.

Con el respaldo de los partidos políticos con representación parlamentaria, el gobierno uruguayo desarrolló una campaña ante diferentes organismos internacionales donde reafirmaba su estrategia internacional de promoción de inversiones, inclusive si ello implicaría el asumir los costos del corte de los puentes.

Más allá de este diferendo, la diplomacia uruguaya encontró puntos fuertes con el éxito en la negociación de la deuda externa con el FMI tras la crisis económico-financiera del año 2002, y en la jerarquización del rol de Naciones Unidas mediante la intervención en operaciones de mantenimiento de paz en Congo y Haití y participando activamente en los diferentes espacios que promueven una reforma en el Sistema de Naciones Unidas. También cabe destacar el estrechamiento en las relaciones con Venezuela y el apoyo a su ingreso al MERCOSUR, el acercamiento a Palestina y a la República Saharaui, el restablecimiento de las relaciones con Cuba, y el acercamiento a los uruguayos residentes en el exterior del país.

Para Luján (2010):

La idea central es que el gobierno de Uruguay ha adoptado como orientación de su política exterior la búsqueda de una inserción internacional en la diversidad, con proyección simultánea hacia el espacio regional y hacia el mundo, lo que ha sido denominado "regionalismo abierto". El balance entre continuidades, ajustes y cambios en lo que refiere a la política exterior del Uruguay durante la presidencia del Dr. Tabaré Vázquez surge del análisis de los puntos fuertes y las oportunidades de mejora que ha tenido el país en materia internacional. ${ }^{\mathrm{V}}$

Para el investigador, este "regionalismo abierto" orientaría a las relaciones bilaterales y multilaterales en detrimento del acentuado pro-americanismo de los anteriores gobiernos.

\section{La política exterior de José Mujica y la continuación de una "buena vecindad" en el relacionamiento con Brasil}

La llegada al poder de los gobiernos progresistas determinó un nuevo escenario de regionalismo sudamericano que se plasmó en el relanzamiento y creación de nuevos espacios de integración regional. Colmado de expectativas en lo que a avances en la integración política y económica se refiere mediante el rechazo - más o menos explicito - al modelo neoliberal de la década de los noventa, estos gobiernos progresistas incorporaron también una nueva agenda social y cultural.

Para el caso de Uruguay, la política exterior del país se debatió entre un modo de inserción en la diversidad y la inclinación hacia un único polo, representado en el acercamiento a Estados Unidos, o en la elección de Sudamérica como espacio de relacionamiento fundamental y prioritario geoestratégicamente donde se potenciaría a Brasil como un actor global en un mundo multipolar (Luján en Quiroga; França, 2010:150-153). 


\title{
LA “ERA PROGRESISTA” EN URUGUAY: ¿CAMBIOS O CONTINUIDADES EN LA POLÍTICA EXTERIOR HACIA BRASIL?
}

\author{
RAFAEL ALVARIZA ALLENDE
}

Con la reelección del Frente Amplio en las elecciones presidenciales de 2009 y la asunción de José Mujica como presidente de los uruguayos el $1^{\circ}$ de Marzo de 2010, varios analistas coinciden con que se procuró dar continuidad a la política exterior desarrollada por su antecesor Tabaré Vázquez.

La plataforma pre-electoral del gobierno de Mujica se encuentra condensada en el "V Congreso Extraordinario Zelmar Michelini" donde nuevamente - al igual que en las elecciones de 2004 - se presenta un capítulo "Uruguay Integrado". Estructurado de manera similar al programa presentado en 2004, este capítulo versa sobre los principios de la política internacional, seguida por aspectos políticos comerciales, los procesos de integración donde se destaca el rol de la UNASUR, la vinculación con los uruguayos en el exterior, la cooperación internacional y el papel del Ministerio de Relaciones Exteriores. Mientras que los principios rectores de la política exterior uruguaya se confunden en primer capítulo de la Carta de las Naciones Unidas, los pilares se refieren a los grandes valores de la izquierda uruguaya (Fernández Luzuriaga, 2010).

Esta plataforma planteaba una estrategia de inserción internacional que privilegiaba al MERCOSUR, pero también al bilateralismo múltiple. En este sentido, se resaltó la necesidad de levantar las barreras intrabloque, de establecer niveles de protección moderados extrabloque y de negociar acuerdos MERCOSUR con terceros países.

El gobierno de Mujica construyó una agenda comercial con metas a corto plazo y a mediano plazo en lo social, sobre una adecuación de estructura institucional y toma de decisiones. Su plataforma de gobierno, preveía también una política monetaria y cambiaria diferenciada, la intervención del sistema financiero público y la regulación de los flujos de capital internacional. Existieron firmes intensiones de evitar la competencia desleal de productos importados mediante el gravamen de impuestos. Bajo el lema "Uruguay abierto al mundo" se configuró una estrategia de desarrollo y de inserción internacional basada en la fórmula "agro+ inteligencia + turismo + logística regional".

En la región, se apoyaron los distintos procesos de integración latinoamericana. Se descartó el ingreso a la Alianza Bolivariana para los Pueblos de Nuestra América (ALBA), se fomentó la cooperación internacional con la creación de la Agencia Uruguaya de Cooperación Internacional (AUCI) y se consideró a la Unión de Naciones Sudamericanas (UNASUR) como la apuesta regional más decisiva junto con el Consejo Sudamericano de Defensa y el Banco del Sur, como espacios de negociación claves en energía, riquezas naturales y renegociación de la deuda externa.

Han sido también iniciativas características de su política exterior, el fortalecimiento del cuerpo diplomático, el reconocimiento de la responsabilidad del Estado ante crímenes de lesa Humanidad - él de mayor repercusión fue el llevado a cabo a partir de la conocida sentencia de la Corte Interamericana de Derechos Humanos por el "Caso Gelman vs. Uruguay" en 2010 - y el discurso en foros internacionales sobre capitalismo, consumo, medioambiente, el papel del hombre y la felicidad. Esto determinó una proyección de la imagen del presidente Mujica a escala global; proyección esta que se ha fortalecido con la 


\section{LA “ERA PROGRESISTA” EN URUGUAY: ¿CAMBIOS O CONTINUIDADES EN LA POLÍTICA EXTERIOR HACIA BRASIL?}

RAFAEL ALVARIZA ALLENDE

adopción de reformas liberales como la despenalización del aborto, el matrimonio igualitario y la regulación del consumo de marihuana en el país.

Con relación a Argentina, el gobierno de José Mujica fortaleció el diálogo teniendo como consecuencia la reafirmación del papel de la Comisión Administradora del Río Uruguay (CARU) y de la Comisión Administradora del Río de la Plata (CARP) y el relanzamiento de comités binacionales de frontera. Asimismo, se mantuvieron reuniones con ambientalistas argentinos y no se autorizó a la empresa Montes del Plata a establecerse en Fray Bentos. Se sucedieron entrevistas con la presidenta Cristina Fernández de Kirchner y se dieron muestras de colaboración internacional, como por ejemplo, con el apoyo de la candidatura del ex Presidente Néstor Kirchner a la Secretaria de UNASUR y la reafirmación de la soberanía de Argentina sobre las Islas Malvinas, Georgias del Sur y Sandwich del Sur.

Por su parte, Argentina, entre otras iniciativas, eliminó las trabas impuestas en el Fondo de Convergencia Estructural del MERCOSUR (FOCEM) al proyecto de interconexión eléctrica con Brasil. A su vez, los ambientalistas levantaron los cortes de puentes binacionales. Sin embargo, la discordia respecto de un aumento en la producción de las plantas procesadoras de celulosa y las dificultades en el diálogo comercial continúan tensionando, por momentos, el relacionamiento bilateral.

Podremos señalar también como otras particularidades de su política exterior, la presencia marcada en la Organización Mundial del Comercio (OMC), el relacionamiento con disidentes cubanos, la colaboración con la Organización para la Cooperación y el Desarrollo Económico (OCDE) para la transparencia fiscal, el intento de relanzar el URUPABOL, la diversificación de sus mercados con Asia y el rechazo a la firma de un Tratado de Libre Comercio con Estados Unidos.

En referencia a la relación bilateral con Brasil, podemos afirmar que están especialmente signadas por la vecindad. En el caso uruguayo esto representa un permanente desafío teniendo en cuenta la extensión, población y poder desproporcional con el que cuenta Brasil. Desde principios de siglo XX, las relaciones entre ambos países han sido de cordialidad y cooperación. Esta "buena vecindad" ha sido clara durante el "período batllista" (1904-1933), el retorno a la democracia (1973-1958/1964-1985), y en la década del noventa con la firma del Tratado de Asunción (1991) que dio origen al MERCOSUR. Sin embargo, con los gobiernos progresistas de Luis Ignácio Lula da Silva (2003-2010) y Dilma Rousseff en Brasil (2011-2014), y Tabaré Vázquez (2005-2010) y José Mujica (2010-2015) en Uruguay, las relaciones bilaterales han experimentado una renovación en la cooperación y una diversificación de agenda.

En este contexto, podremos afirmar que Brasil ha desenvuelto una política exterior con el claro objetivo de alcanzar el desarrollo nacional y de convertirlo en recurso de poder económico, industrial y militar - poniendo al servicio del interés nacional el progreso tecnológico, la cooperación internacional, las inversiones, la ampliación de los mercados - así como también la obtención del reconocimiento internacional como "potencia regional" y el establecimiento de "alianzas estratégicas" con el resto del mundo. 


\title{
LA “ERA PROGRESISTA” EN URUGUAY: ¿CAMBIOS O CONTINUIDADES EN LA POLÍTICA EXTERIOR HACIA BRASIL?
}

\author{
RAFAEL ALVARIZA ALLENDE
}

La elección de Lula da Silva (2003-2010) del Partido dos Trabalhadores (PT) promovió una inserción internacional interdependiente (Cervo; Bueno, 2012). Su gobierno incentivó la internacionalización de las empresas brasileñas, promovió la integración regional en América del Sur - siendo la UNASUR su apuesta más importante en 2011 -, diversificó las relaciones con árabes y africanos, fortaleció la imagen de Brasil como actor internacional teniendo como objetivo principal la obtención de un asiento permanente en el Consejo de Seguridad de Naciones Unidas.

Con la asunción de los gobiernos progresistas en Brasil y Uruguay, se estrechan notablemente las relaciones bilaterales. Un mes después de asumir la Presidencia de la República, en Abril de 2005 Tabaré Vázquez eligió a Brasilia como su primera visita oficial al exterior. Ese fue el puntapié inicial para una serie de visitas recíprocas entre gobernantes progresistas.

En Marzo de 2006, cuando Lula da Silva recibió nuevamente al Presidente uruguayo en Brasilia, el mandatario brasileño expresó:

As profundas afinidades entre nossos dois países e governos exigem que avancemos
nos grandes projetos de integração física e produtiva de modo a garantir o
desenvolvimento solidário de nossos povos. Para cumprir esse objetivo determinei a
realização de reuniões em nível ministerial, para tratar de todos os aspectos das
relações com o Uruguai [...] No Brasil, temos acompanhado com grande interesse e
entusiasmo as realizações de seu governo. A retomada vigorosa do crescimento
econômico com justiça social é também o alvo maior de meu governo [...] A
franqueza e o espírito construtivo com que conversamos, os entendimentos a que
chegamos, me dão uma certeza. Esta sua visita ao Brasil marcará uma nova fase no
diálogo entre nossos países e na realização do potencial de cooperação entre nossos
dois povos . ${ }^{\text {II }}$

De esta manera, el relacionamiento con el Brasil fue uno de los temas que formaron parte del debate acerca de la agenda internacional del primer gobierno progresista. En este sentido, Luján expresa (2010):

Las relaciones con el Brasil de Lula han sido excelentes, más allá de inconvenientes
comerciales en nuestras fronteras, todos ellos subsanables. Las frecuentes visitas del
asesor personal del Presidente brasileño en materia internacional, el Dr. Marco
Aurelio García al Uruguay son un indicador de la muy buena relación existente entre
dos gobiernos afines, afinidad inexistente con el anterior gobierno colorado. Sin
embargo, tan buen relacionamiento no se ha traducido en una acción de liderazgo
regional brasileño que permitiera mejorar las debilitadas relaciones con Argentina
VII.

En el gobierno de José Mujica las relaciones bilaterales continuaron el curso de la "buena vecindad" y la cooperación establecida a partir de los gobiernos Da Silva-Vázquez. En este sentido, la elección de Montevideo en Mayo de 2011 por la presidenta Dilma Rousseff como destino de uno de sus primeros viajes oficiales al exterior desde su asunción y la posterior definición de las relaciones con Uruguay como "estratégicas", fue una clara señal que la diplomacia uruguaya supo aprovechar.

La política exterior de Dilma Rousseff se podría definir a partir de la continuidad de los principales objetivos en la esfera internacional postulados en esta área por la gestión Lula da Silva. Sin embargo, la diplomacia de Rousseff procura estos objetivos con menor

Cadernos do Tempo Presente, n. 24, jun./jul. 2016, p. 110-119| http://www.seer.ufs.br/index.php/tempo 


\title{
LA “ERA PROGRESISTA” EN URUGUAY: ¿CAMBIOS O CONTINUIDADES EN LA POLÍTICA EXTERIOR HACIA BRASIL?
}

RAFAEL ALVARIZA ALLENDE

activismo y mayores limitaciones externas, caracterizándose por un ajuste en medio de la continuidad; mientras que Lula se consolidó como líder político a partir de sus dotes carismáticos, Rousseff se destacó en la administración pública por su capacidad técnica y gestora (Conte Cornetet, 2014).

En una reunión con empresarios y altas autoridades del gobierno uruguayo, el presidente Mujica destacó la importancia de un estrecho relacionamiento con el Brasil al expresar:

\begin{abstract}
Estamos al lado de una potencia excelente que se llama Brasil, y está viviendo un momento histórico, que tiene un gobierno afín. Que torpes nosotros si no sabemos aprovechar esa posibilidad con los de arriba, porque aparte de todo este Brasil de hoy tiene voluntad política. Y antes, no [...] Brasil tiene necesidad de jugar como potencia y como tal necesita influencia de carácter político, es la potencia emergente del Atlántico Sur. Brasil es un país de casi 200 millones de habitantes, con una reserva petrolífera enorme pero sobre todo, tiene la mayor reserva de energía hidroeléctrica disponible en el mundo [...] es hora de hablar en portugués despacio y en español despacio, a partir de ahí nos entendemos. Y no entrar en los nacionalismos pequeños de cotorro que nos estropean el otro verdadero nacionalismo que es la construcción de la posibilidad de esta América VIII.
\end{abstract}

\section{Una nueva dinámica de cooperación e integración bilateral}

En su Teoría de la Interdependencia y de los Regímenes Internacionales, Robert O. Keohane y Josep S. Nye (1989) sostienen que los Estados son interdependientes. Donde existe intercambio, hay necesariamente interdependencia. En los sistemas políticos internacionales, los Estados son las unidades de mayor importancia que "buscan maximizar sus intereses y poder". La interdependencia puede ser fuente de poder, entendida como la habilidad de un actor para lograr que sus pares tomen determinadas acciones que de otra forma no lo harían, controlando potencialmente sus recursos y resultados. La interdependencia simple indica que los Estados a través de su acción nacional unilateral, no reúnen la capacidad suficiente para alcanzar óptimos resultados. En cambio, la interdependencia compleja se caracteriza por la existencia de múltiples canales que conectan las sociedades en sus relaciones interestatales, transgubernamentales y transnacionales; por una agenda de variados temas no jerarquizados (económicos, energéticos, ecológicos, culturales, etc.) y no supeditados a la seguridad militar; y por la ausencia del uso de la fuerza. En la construcción de una agenda de integración y cooperación hacia el Brasil, la interdependencia compleja parece jugar un papel de relevancia.

En materia de política exterior se perciben cuatro dimensiones claramente identificables. En una primera dimensión se identifica a la agenda global; en una segunda dimensión los temas referidos a los espacios regionales de integración conforman la agenda regional; finalmente, en la tercera dimensión se analizan las relaciones bilaterales con énfasis en el nuevo paradigma de cooperación e integración de estos Estados proponen.

La dimensión multilateral del relacionamiento ambos países responde claramente a dos iniciativas: la disposición de Brasil de proyectarse regionalmente y de asumir los costos, y el reconocimiento uruguayo al liderazgo de Brasil. 


\title{
LA “ERA PROGRESISTA” EN URUGUAY: ¿CAMBIOS O CONTINUIDADES EN LA POLÍTICA EXTERIOR HACIA BRASIL?
}

\author{
RAFAEL ALVARIZA ALLENDE
}

\begin{abstract}
El papel privilegiado de Brasil en el escenario internacional reforzó su liderazgo en la región, concomitantemente con el desarrollo de una política exterior que mostró la clara intención de asumir los costos por este liderazgo. Ello se debe al cambio en el modelo de desarrollo que Brasil inició a partir del gobierno de Lula Da Silva, donde se prioriza el desarrollo mediante la internacionalización de la economía. Aquí las iniciativas bilaterales de integración aparecen como una oportunidad de esta internacionalización (Mallmann; Marques, 2013: 11). En este contexto cabe recordar las claras asimetrías existentes entre Uruguay y Brasil - y de este gigante con el resto de América Latina - y que se han agudizado en la última década. Estas profundas asimetrías, también evidentes en el MERCOSUR y otros espacios de integración, fortalecieron una orientación de Brasil hacia una política exterior que privilegie también el bilateralismo con sus vecinos sudamericanos.
\end{abstract}

A su vez, cabe destacar que con relación a Brasil, Uruguay viene desarrollando una política de reconocimiento de su liderazgo. Prueba de ello, es el apoyo uruguayo a los posicionamientos de Brasil en diversos foros internacionales. Un ejemplo es la necesidad de reforma de las Naciones Unidas y el nombramiento de Brasil como Miembro Permanente del Consejo de Seguridad. En el seno de la ONU, ambos países han colaborado estrechamente en la Misión de Naciones Unidas para la Estabilidad en Haití (MINUSTAH).

En comunicado de prensa del 21 Octubre de 2013, en ocasión de la primera visita oficial del Canciller brasileño Luiz Alberto Figueredo a Uruguay, se remarcó que:

Uruguay y Brasil coincidieron en "seguir los compromisos con el multilateralismo y
con Haití, pero también cumplir con los compromisos asumidos en el marco de la
UNASUR y del propio mandato de ONU, de reducción progresiva de efectivos".
Allí, el Canciller uruguayo, Luis Almagro señaló que se trataron otros temas de
interés global: "las Naciones Unidas, la situación en Medio Oriente, asuntos de
cooperación y temas comerciales en general, así como en el avance para lograr
consensos regionales en torno a una candidatura en el marco de la Unasur"IX.

En la OMC ambos países abogan por el desarrollo de un sistema multilateral de comercio. También allí, existieron consensos en las negociaciones de la Ronda de Doha sobre la retirada de barreras proteccionistas a los productos agrícolas. Otros posicionamientos conjuntos se han demostrado, entre otros, con respecto al establecimiento de relaciones con Palestina y reconocimiento de autodeterminación de su pueblo, así como la condena a la violación de derechos humanos en varios países del mundo, como el caso de Irán. Recientemente la propuesta uruguayo-brasileña llevada a la OEA donde se respaldó al gobierno argentino en la contienda que mantiene con los fondos financieros especuladores acreedores de deuda pública, ha demostrado la solidez en la construcción de una agenda global con coincidencias.

También, Uruguay ha apoyado diversas candidaturas de funcionarios brasileños para la ocupar las direcciones de algunos foros internacionales. Tal es el caso de la candidatura de Jose Graziano da Silva al cargo de Director General de la Organización Mundial para la Agricultura (FAO) para el período 2012-2015 y la de Roberto Azevedo como Director General de la OMC. En contrapartida, Brasil apoyó la candidatura de Uruguay como Miembro No Permanente del Consejo para el período 2016-2017. En este sentido, los gobiernos de Rousseff y Mujica han demostrado mayor sintonía que los anteriores gobiernos

Cadernos do Tempo Presente, n. 24, jun./jul. 2016, p. 110-119| http://www.seer.ufs.br/index.php/tempo 


\section{LA “ERA PROGRESISTA” EN URUGUAY: ¿CAMBIOS O CONTINUIDADES EN LA POLÍTICA EXTERIOR HACIA BRASIL?}

RAFAEL ALVARIZA ALLENDE

de Lula da Silva y Tabaré Vázquez, cuando estos lanzaron candidatos propios para la OMC y cuando Uruguay no apoyó al candidato brasileño a la presidencia del Banco Interamericano de Desarrollo (BID).

Con respecto a la dimensión regional presente en el relacionamiento de ambos países, interesa especialmente la referida al MERCOSUR como espacio privilegiado de integración regional.

En lo que hace respecto a la agenda regional, el apoyo al fortalecimiento de la Unión de Naciones Latinoamericanas (UNASUR), de las Comunidad de Estados Latinoamericanos y Caribeños (CELAC) así como la reactivación de la Zona de Paz y Cooperación del Atlántico Sur (ZOPACAS), son claras demostraciones de cooperación. Sin embargo, es el MERCOSUR la iniciativa regional más importante que han impulsado ambos Estados y como espacio privilegiado de integración.

A su vez, fue marcada por la suspensión de Paraguay y por el ingreso de Venezuela al bloque. El juicio político que depuso al entonces presidente Fernando Lugo en Junio de 2012 representó uno de los desafíos políticos de mayor trascendencia para la región. Considerado un acto de ruptura democrática en aquel país, Paraguay fue suspendido del MERCOSUR y de la UNASUR. Además de las medidas institucionales, los presidentes de Uruguay y de Brasil hicieron fuertes declaraciones al respecto; mientras que Mujica clasificó el episodio de un "golpe de Estado parlamentario" (Uruguay, 2012, 5 de Junio), Itamaraty condenó la destitución de Lugo afirmando que no se contempló el derecho de defensa, comprometiendo a la democracia, condición esencial para la integración regional (Brasil, 2012, 23 de Junio). Paralelamente, en julio de 2012 se produjo el ingreso de Venezuela como miembro pleno del bloque, ocasión donde nuevamente los presidentes coincidieron en defender la importancia geopolítica y económica del país caribeño. Ambos países han saludado también el ingreso de Bolivia - ya ratificado por Uruguay y Venezuela - al bloque y que estiman se consolidará próximamente.

En un comunicado conjunto del 30 de Mayo de 2011, los presidentes Mujica y Rousseff reafirmaron que el proyecto integracionista es:

\footnotetext{
Essencial como instrumento na melhora da qualidade de vida de seus povos e para a inserção internacional do bloco, destacando seu papel estratégico na integração política, social, econômica e comercial, e identificando a necessidade da plena implementação da união aduaneira em curto prazo ${ }^{\mathrm{X}}$.
}

A pesar de estas coincidencias a nivel regional, cabe resaltar algunas diferencias a entre ambas diplomacias. En 2006, el gobierno brasileño rechazó las negociaciones emprendidas por el gobierno de Tabaré Vázquez para la concreción de un Tratado de Libre Comercio (TLC) con Estados Unidos y en claro debilitamiento del MERCOSUR. Aunque la firma de este TLC con la potencia norteamericana no se llegó a concretizar, este acercamiento fue interpretado por varios analistas como una respuesta de Uruguay ante la neutralidad de Brasil - en el diferendo que lo enfrentaba con Argentina por la instalación de plantas de celulosa - y su incapacidad de actuar como mediador.

En julio de 2012, el gobierno de Rousseff criticó severamente la aceptación de Uruguay de ingresar como observador a la Alianza del Pacífico (Uruguay, 25 de Julio de 


\section{LA “ERA PROGRESISTA” EN URUGUAY: ¿CAMBIOS O CONTINUIDADES EN LA POLÍTICA EXTERIOR HACIA BRASIL?}

RAFAEL ALVARIZA ALLENDE

2012), entendiendo que ha dado fuertes señales a Uruguay con la construcción de una solida agenda bilateral (La Diaria, 2012, $1^{\circ}$ de Octubre).

En este sentido, la debilidad del MERCOSUR se manifestó en un patrón de relacionamiento regional adepto al bilateralismo y poco simpatizante a los acuerdos institucionales multilaterales profundos. La reducida institucionalización del MERCOSUR y de otras instancias intergubernamentales tales como la UNASUR confirma en cierta medida esta premisa. A pesar de que Brasil en política exterior desarrolle un discurso que apunta al comportamiento multilateral como prioridad, delante de los costos reducidos de las negociaciones bilaterales con sus vecinos, esta vía parece haber sido la privilegiada por Itamaraty (Alvariza, et al., 2014). A pesar de ello, cabe destacar que diferentemente de otras experiencias de integración que sucumbieron de cara al colapso económico y comercial, el MERCOSUR - y en términos más amplios, la integración sudamericana - continúa agregando temas nuevos en sus agendas correspondientes.

Como se ha dicho, en el caso de las relaciones uruguayo-brasileñas, es notorio el fortalecimiento de las agendas bilaterales a partir de la llegada al poder de los gobiernos progresistas. Sin embargo, fue quizás el 31 de Julio de 2012 que estas relaciones se estrecharon aún más con el inicio de las negociaciones bilaterales que tuvieron como resultado el establecimiento de una asociación estratégica con base en un "nuevo paradigma" de integración bilateral, materializado en la creación del "Grupo de Alto Nivel BrasilUruguay (GAN). El GAN fue creado para ejecutar el "Plan de Acción para el Desarrollo Sustentable y la Integración" cuyo objetivo es la intensificación de diversos proyectos de integración así como la complementación productiva y la cooperación. Este plano abrió espació también para la asociación entre actores públicos y privados que manifiesten su interés en la cooperación bilateral (Brasil, 31 de Julio de 2012).

Desde el punto de vista uruguayo, este nuevo paradigma se inserta en la estrategia del gobierno frenteamplista que continúa privilegiando el MERCOSUR como plataforma de inserción internacional (Alvariza, 2013), aunque también privilegie el bilateralismo múltiple. Bajo el lema "Uruguay abierto al mundo" reciben especial atención las áreas vinculadas a la logística regional, a la agropecuaria y al turismo, así como la apertura y diversificación de mercados.

Un año luego de la consolidación de un "nuevo paradigma de integración bilateral" entre Brasil y Uruguay, los Ministros de Relaciones Exteriores, Antonio Patriota y Luis Almagro, celebraron el 9 de julio de 2013 en Brasilia la $1^{\circ}$ Reunión Plenaria del Grupo de Alto Nivel Brasil-Uruguay (GAN). Este encuentro entre altas autoridades tuvo como consecuencia la aprobación del "Plan de Acción para el Desarrollo Sustentable y la Integración Uruguay-Brasil".

El "Plano de Acción" bilateral establece objetivos generales y específicos, la división de tareas y responsabilidades, la identificación de puntos focales en ambos gobiernos, así como plazos para la consolidación de la integración bilateral. Con este propósito, se conformaran seis "subgrupos de trabajo" o "áreas" donde se trabajarán iniciativas consideradas prioritarias. Integración productiva; Ciencia, tecnología e innovación; Comunicación e información; Integración de infraestructura de transportes; Libre circulación

Cadernos do Tempo Presente, n. 24, jun./jul. 2016, p. 110-119| http://www.seer.ufs.br/index.php/tempo 


\section{LA “ERA PROGRESISTA” EN URUGUAY: ¿CAMBIOS O CONTINUIDADES EN LA POLÍTICA EXTERIOR HACIA BRASIL?}

RAFAEL ALVARIZA ALLENDE

de bienes y servicios; y Libre circulación de personas; conforman los subgrupos que deberán proseguir sus actividades y reuniones de forma independiente. El GAN es el responsable por el acompañamiento de estos subgrupos y por la evaluación de los avances registrados, con la clara intensión de dar cumplimiento a los objetivos establecidos en el "Plano de Acción", el cual también podrá ser modificado y actualizado con nuevos proyectos (Uruguay, 2013, 21 de Octubre). En este contexto, cabe destacar las numerosas reuniones entre ministros y demás representantes gubernamentales en ambos países.

En integración productiva, se viene desarrollando proyectos de cooperación energética, principalmente en energía eólica. El intercambio de energía eléctrica a través de la conversora instalada en Rivera (72 MW), la inversión conjunta de UTE y ELECTROBRAS en la creación de un parque de energía eólica y la construcción de una línea de transmisión eléctrica de $500 \mathrm{KW}$ entre las ciudades de San Carlos (Uruguay) y Candiota (Brasil), están entre las negociaciones destacadas. En lo referido a ciencia, tecnología e innovación se registra un mecanismo de cooperación bilateral que agrupa los temas de biotecnología, nanotecnología, medio ambiente, biomedicina y tecnologías de la información y comunicación (TIC's). En este sentido, se proyecta la creación de una plataforma digital para la formación de recursos humanos en TIC's y la creación del Centro Binacional de Tecnologías de la Información y de la Comunicación. Fue previsto también el ingreso de Uruguay al Centro Brasil-Argentina de Biotecnología (CBAB) (Alvariza, 2013).

Sobre la cooperación en ciencia y tecnología, la presidenta Dilma afirmó:

El presidente Mujica y yo decidimos también expandir los horizontes temáticos de
nuestra agenda bilateral, creamos un mecanismo nuevo para coordinar los
esfuerzos de cooperación en el campo de la ciencia y tecnología. Vamos
apoyar proyectos de desarrollo conjunto en el campos de biotecnología,
la nanotecnología, de tecnología de la información XI.

Con la decisión de Uruguay de adoptar la norma nipo-brasileña de TV digital (ISDBT) se ha registrado importantes esfuerzos en comunicación e información, donde se está trabajando temas vinculados a contenidos digitales creativos, aplicaciones interactivas, democratización del acceso a internet y telecomunicaciones en general. También se están desarrollando proyectos para la interconexión de redes y la atención en las áreas de frontera entre las empresas estatales ANTEL y TELEBRAS (Brasil, 31 de Julio de 2012).

Otro tema donde se registra avances es en la cooperación en defensa, asuntos espaciales y estadísticas a partir del interés de incluir a la industria naval en iniciativas binacionales, así como en el intercambio de experiencias, en las operaciones conjuntas de defensa, en la ejecución de proyectos conjuntos entre el Instituto Nacional de Investigaciones Espaciales (INPE) y la Dirección Nacional de Meteorología (DNM), además de la cooperación ente el Instituto Brasilero de Geografía y Estadística (IBGE) y el Instituto Nacional de Estadística (INE) (Alvariza, 2013).

Entre los principales avances en Integración de infraestructura de transportes se identifican el establecimiento de un cronograma preliminar que establece para 2017 la finalización de la construcción de un nuevo puente sobre el río Yaguarón dedicado al tránsito de cargas pesadas y la restauración del Puente Internacional Barón de Mauá que une a las ciudades de Yaguarón (Brasil) y Rio Branco (Uruguay), buscando beneficiar al turismo y el

Cadernos do Tempo Presente, n. 24, jun./jul. 2016, p. 110-119| http://www.seer.ufs.br/index.php/tempo 


\section{LA “ERA PROGRESISTA” EN URUGUAY: ¿CAMBIOS O CONTINUIDADES EN LA POLÍTICA EXTERIOR HACIA BRASIL?}

RAFAEL ALVARIZA ALLENDE

transito local. Reciben atención también la interconexión ferroviaria entre las ciudades de Montevideo, Rivera, Santana do Livramento y Cacequi como vía para facilitar el comercio terrestre, y cuyo proyecto recibirá en parte financiamiento del FOCEM. Vale destacar también la Hidrovía Brasil-Uruguay, que posibilitará un sistema de transporte fluvial moderno de cargas y pasajeros, ofreciendo una alternativa de bajo costo para el transporte multimodal; este tema viene siendo trabajado también por las Comisiones Mixtas de la Cuenca de la Laguna Merín y del Rio Cuareim. También está presente en la agenda la construcción de un puerto de aguas profundas en el departamento uruguayo de Rocha así como la internacionalización del Aeropuerto de Rivera, donde Uruguay esta previendo incorporar otros países socios ${ }^{\mathrm{XII}}$.

En lo que se refiere a la libre circulación de bienes y servicios, se destacan la negociación de la aplicabilidad del "Acuerdo de Complementación Económica" (ACE n²) suscrito por ambos países (ALADI, 2013). En este sentido, se prevén mecanismos bilaterales para medidas sanitarias y fitosanitarias, reglamentación técnica y procedimientos de evaluación de conformidad, así como también el compromiso de armonizar trámites aduaneros y mecanismos para dirimir divergencias comerciales. Para alcanzar estos objetivos, se creó la Comisión de Comercio Bilateral (CCB) y se ha tenido la colaboración del Instituto de Investigación Económica Aplicada (IPEA) de Brasil. Recientemente, Uruguay ha manifestado la intensión de liberalizar el tránsito de algunos bienes y servicios con Brasil en un futuro próximo XIII.

A su vez, en lo que se refiere a la libre circulación de personas, se destacan los acuerdos obtenidos en materia de residencia, derechos laborales y políticas educativas y culturales, con claras ganancias para la población en general, principalmente la localizada en áreas de frontera. La implementación de la Agenda Binacional de Cooperación y Desarrollo Fronterizo ocurre en una región cuyas características han sido construidas históricamente desde la formación de las poblaciones en los dos países. Los intercambios económicos, los movimientos migratorios, los conflictos políticos y las influencias culturales de ambos lados de la línea fronteriza contribuyeron a la génesis de una sociedad con perfil propio. La demarcación territorial entre los dos Estados no fue obstáculo para la formación de una "frontera abierta" entendida como un espacio de múltiples interacciones entre sus habitantes. Con la aprobación, en 2002, de la Nueva Agenda de Cooperación y Desarrollo Fronterizo, fue puesto en práctica un plan de acción para el desarrollo integrado de la región.

Responsable por la articulación de políticas integradas y por las negociaciones de instrumentos jurídicos innovadores, esta agenda beneficiará a aproximadamente $800 \mathrm{mil}$ personas a lo largo de $1.069 \mathrm{~km}$ de frontera. Entre los principales acuerdos se destaca el "Acuerdo sobre permiso de residencia, estudio y trabajo para los nacionales fronterizos uruguayos y brasileños" (2010) a través del "Documento especial de fronterizo" expedido por el Departamento de Policía Federal de Brasil y por la Dirección Nacional de Migraciones de Uruguay. El presente acuerdo incluye también el "Ajuste complementario del Acuerdo sobre permiso de residencia, estudio y trabajo para los nacionales fronterizos uruguayos y brasileños para prestación de Servicios de Salud" en sistemas públicos o contrataos entre personas jurídicas y físicas, situadas en las localidades fronterizas (Alvariza, 2013). Otras iniciativas se refieren al "Proyecto de Saneamiento Integrado Acegua - Aceguá" con financiamiento del Fondo de Convergencia Estructural del MERCOSUR (FOCEM) aprobado en 2005 por el 


\section{LA “ERA PROGRESISTA” EN URUGUAY: ¿CAMBIOS O CONTINUIDADES EN LA POLÍTICA EXTERIOR HACIA BRASIL?}

RAFAEL ALVARIZA ALLENDE

bloque con el objetivo de disminuir la asimetría entre los miembros, y una variada oferta de cursos binacionales impartida por centros educativos públicos de la región.

El objetivo central de esta estrategia es la promoción del desarrollo integral de la línea de frontera común, que reconoce a Rio Grande do Sul como sede alternativa de las reuniones entre representantes de los gobiernos centrales. En el encuentro de Octubre de 2013 en Montevideo entre los Cancilleres Luiz Alberto Figueiredo y Luis Almagro, el Canciller brasilero enfatizó el objetivo de que esta integración bilateral tenga un carácter ejemplar en la región (Uruguay, 2013, 21 de Octubre).

\section{Consideraciones finales}

El advenimiento de la llamada "era progresista" supuso cambios en la política exterior de Uruguay sin entrar en contradicción con los tradicionales pilares que le han dado sustento. Estos cambios encuentran explicación, en parte, a partir de un análisis de la dimensión internacional presente en los programas de gobierno de la izquierda uruguaya y su adaptación a los problemas propios del orden internacional imperante.

La llegada al poder de Tabaré Vázquez en 2005 pretendió poner en práctica un proyecto de inserción internacional activo que, en un principio, procuró priorizar los procesos de integración regionales y fortalecer la bilateralidad con sus principales socios - Argentina y Brasil - pero que finalmente se orientó al pragmatismo en un contexto de regionalismo abierto y de inserción en la diversidad. La reelección del gobierno frenteamplista con José Mujica en 2009, proyectó una continuidad a la política exterior de su antecesor pero que adquirió identidad propia, cuando se procuraron alternativas que fortalecieran el diálogo con Argentina, por ejemplo.

Delante de un nuevo escenario de regionalismo sudamericano y ante las vicisitudes de una interdependencia compleja, ambos gobiernos progresistas favorecieron particularmente el acercamiento a Brasil. Una serie de visitas entre mandatarios de ambos países fue una clara demostración del nacimiento de una nueva agenda de integración y cooperación que asumió claramente tres dimensiones: multilateral, regional y bilateral. Mientras que en la dimensión multilateral el apoyo a Brasil en su proyección internacional resulta clave, en la dimensión regional se privilegia al MERCOSUR como espacio de integración. Sin embargo, es la dimensión bilateral la demostración de mayor relevancia acerca un cambio en la política exterior de Uruguay hacia su vecino norteño, siendo la creación del "Grupo de Alto Nivel Brasil-Uruguay" la materialización de un "nuevo paradigma" en el relacionamiento de ambos países.

En este sentido, la puesta en práctica de una nueva agenda multidimensional favorece que el análisis de la política exterior nacional hacia Brasil se realice desde una perspectiva que contemple cambios y continuidades desde una mirada no antagónica sino complementaria.

\section{Referencias bibliográficas}

Cadernos do Tempo Presente, n. 24, jun./jul. 2016, p. 110-119| http://www.seer.ufs.br/index.php/tempo 


\section{LA “ERA PROGRESISTA” EN URUGUAY: ¿CAMBIOS O CONTINUIDADES EN LA POLÍTICA EXTERIOR HACIA BRASIL?}

RAFAEL ALVARIZA ALLENDE

Asociación Latinoamericana de Integración (ALADI) (2013). Acuerdo de Complementación Económica $N^{\circ} 2$ celebrado entre la República Federativa del Brasil y la República Oriental del Uruguay - Septuagésimo Primer Protocolo Adicional. Disponible en: http://www.aladi.org/nsfaladi/textacdos.nsf/b3198303bf8b318403256fc50052d176/367c9d8d 126de1d7032570e3004b1b78?OpenDocument

Alvariza Allende, R. (2012, 7 de Mayo). Positivas repercusiones de la visita de Mujica a Brasil. CEIRI Newspaper. Disponible en: Disponible en: http://www.jornal.ceiri.com.br/positivas-repercusiones-de-la-visita-de-mujica-a-brasil/

Alvariza Allende, R. (2013). Uruguai-Brasil: cooperação e integração nos governos de Mujica e Rousseff. Mundorama: Divulgação Científica em Relações Internacionais do Instituto de Relações Internacionais da Universidade de Brasília - iREL-UnB, 69, p. 1.

Alvariza Allende, R.; Mallmann, M. I.; Schneider Marques, T.C. (2014). Relações uruguaiobrasileiras: uma breve aproximação das atuais agendas de cooperação. En: Rückert, A. (Ed.) Integração regional, fronteiras e globalização no continente americano. Porto Alegre: Editora Compasso Lugar-Cultura \& Imprensa Livre. Manuscrito no publicado.

Ministério das Relações Exteriores do Brasil (2006). Discurso do Presidente da República, Luiz Inácio Lula da Silva por ocasião da visita do Presidente do Uruguai, Tabaré Vázquez. Disponible en: http://www.itamaraty.gov.br/sala-de-imprensa/discursos-artigos-entrevistas-eoutras-comunicacoes/presidente-da-republica-federativa-do-brasil/15969536046-discurso-dopresidente-da-republica-luiz-inacio/

Brasil, Ministério das Relações Exteriores do (2011, 30 de Mayo). Atos assinados por ocasião da visita da Presidenta Dilma Rousseff ao Uruguai. Disponible en: http://www.itamaraty.gov.br/index.php?option=com content $\&$ view=article $\& i d=2623 \&$ catid $=$ 42: notas\&Itemid=280\&lang=pt-br

Brasil, Presidência da Republica do. (2011, 30 de Mayo). Declaração à imprensa da Presidenta da República, Dilma Rousseff, em conjunto com o Presidente da República do Uruguai, José Mujica. Montevidéu, Uruguai. 30 de maio de 2011. Disponible en: http://www2.planalto.gov.br/imprensa/discursos/declaracao-a-imprensa-da-presidenta-darepublica-dilma-rousseff-em-conjunto-com-o-presidente-da-republica-oriental-do-uruguaijose-mujica

Brasil, Ministério das Relações Exteriores do. (2012, 23 de Junio). Situação no Paraguai, nota $\mathrm{n}^{\mathrm{o}}$ 155. Disponible en: http://www.itamaraty.gov.br/sala-de-imprensa/notas-aimprensa/situacao-no-paraguai/

Cervo, A. L.; Bueno, C. (2012). História da política exterior do Brasi (4 ${ }^{a}$ ed.). Brasilia: Editora Universidade de Brasilia - UnB.

Clemente, I. (2004). La política exterior del primer gobierno de izquierda en Uruguay. Nordic journal of Latin American and Caribbean Studies, vol. 34 (1-2), pp. 323-351. 


\section{LA “ERA PROGRESISTA” EN URUGUAY: ¿CAMBIOS O CONTINUIDADES EN LA POLÍTICA EXTERIOR HACIA BRASIL?}

RAFAEL ALVARIZA ALLENDE

Conte Cornetet, J. M. (2014). A política externa de Dilma Rousseff: contenção na continuidade. Revista Conjuntura Austral, vol. 5 (24), pp. 111-150.

Ferro Clérico, L., Fernández Luzuriaga, W. y Hernández Nilson, D. (2006). La estrategia de inserción internacional de Uruguay en el gobierno del Frente Amplio. Revista Uruguaya de Ciencia Política, vol. 15 (1), pp. 129-150.

Fernández Luzuriaga, W. (2005). La Política Exterior del Uruguay en las Elecciones Nacionales de 2004. Serie Documentos de Trabajo $n^{\circ}$ 66, Unidad Multidisciplinaria, Facultad de Ciencias Sociales, Universidad de la República.

Fernández Luzuriaga, W. (2010). El presidente electo en la transición, trazos de la política exterior del gobierno de José Mujica. Serie Documentos de Trabajo $n^{\circ} 78$, Unidad Multidisciplinaria, Facultad de Ciencias Sociales, Universidad de la República.

Garcé, A.; Yaffé, J. (2005). La era progresista. Montevideo: Fin de Siglo.

Keohane, R. O. y Nye, J. S. (1989). Power and Independence. Nueva York: Harper Collins Publishers.

La Diaria (2012, $1^{\circ}$ de Octubre). De primera mano. Disponible en: http://ladiaria.com.uy/articulo/2012/10/de-primera-mano/.

Mallmann, M. I.; Schneider Marques, T. C. (2013). A crise do multilateralismo e o Mercosul: uma lógica de integração? En Encontro Nacional da Associação Brasileira de Relações Internacionais: Multilateralismo, plurateralismo e a construção da ordem mundial (4: Belo Horizonte: 2013). Trabajos presentados. Belo Horizonte: ABRI.

Observatorio de Política Exterior Uruguaya. Montevideo: Programa de Estudios Internacionales, Facultad de Ciencias Sociales, Universidad de la República. Disponible en: http://observatoriopoliticaexterioruruguaya.wordpress.com/

Pérez Antón, R. (2011). La política exterior uruguaya en el siglo XX. Montevideo: Ediciones de la Plaza.

Quiroga, Y. y França, C. (Org.) (2010). Consenso progresista. Política exterior de los gobiernos progresistas del Cono Sur: convergencias y desafíos. São Paulo: Fundación Friedrich Ebert, Red de Fundaciones Progresistas del Cono Sur.

Russell, R. (1990). Política exterior y toma de decisiones en América Latina. Buenos Aires: Grupo Editor Latinoamericano.

Uruguay, Presidencia de la República Oriental del. (2012, 25 de Junio). Mujica rechazó sanciones económicas ante "golpe de Estado parlamentario" en Paraguay. Disponible en: http://www.presidencia.gub.uy/comunicacion/comunicacionnoticias/mujica-paraguay-golpeestado-parlamentario-canciller-sanciones-economicas-cumbre-mercosur.

Uruguay, Ministerio de Relaciones Exteriores del. (2012). Uruguay aceptó en Perú ingresar a la Alianza del Pacífico como observador. 25 de Julio de Disponible en: Cadernos do Tempo Presente, n. 24, jun./jul. 2016, p. 110-119| http://www.seer.ufs.br/index.php/tempo 


\section{LA “ERA PROGRESISTA” EN URUGUAY: ¿CAMBIOS O CONTINUIDADES EN LA POLÍTICA EXTERIOR HACIA BRASIL?}

RAFAEL ALVARIZA ALLENDE

http://www.presidencia.gub.uy/comunicacion/comunicacionnoticias/mujica-paraguay-golpeestado-parlamentario-canciller-sanciones-economicas-cumbre-mercosur. Consultado el $\underline{02 / 04 / 2014}$

Uruguay, Presidencia de la República Oriental del. (2012, 31 de Julio). Comunicado Conjunto Presidencial: Un nuevo paradigma para la relación Brasil-Uruguay. Disponible en: http://medios.presidencia.gub.uy/jm_portal/2012/noticias/NO_F064/comunicado.pdf

Uruguay, Presidencia de la República Oriental del. (2013,10 de Mayo). Uruguay negocia con Brasil eliminación de la frontera para algunos bienes y servicios. Disponible en: http://www.presidencia.gub.uy/Comunicacion/comunicacionNoticias/porto-vicenciller.

Uruguay, Ministerio de Relaciones Exteriores del. (2013, 21 de Octubre). Encuentro entre Uruguay $\quad$ B $\quad$ Brasil. Disponible en: http://www.mrree.gub.uy/frontend/page?1,inicio,ampliacionppal2,O,es,0,PAG;CONP;1961;11;P;encuentro-entre-uruguay-y-brasil;8;PAG

Van Klaveren, A. (1992). Entendiendo las políticas exteriores latinoamericanas: modelo para armar. Revista de Estudios Internacionales, Instituto de Estudios Internacionales, Universidad de Chile, (98), año XXV, pp. 169-216.

\section{Notas}

\footnotetext{
I Licenciado en Relaciones Internacionales y estudiante de Maestría en Ciencias Humanas Opción Estudios Latinoamericanos por la Universidad de la República (UdelaR, Uruguay). Becario por el Convenio CAPESUdelaR con estancia en el Programa de Postgrado en Ciencias Sociales de la Pontifícia Universidade Católica do Rio Grande do Sul (PUC-RS, Brasil). Investigador colaborador del Programa de Estudios Internacionales de la Facultad de Ciencias Sociales de la UdelaR y del Núcleo de Estudos em Relações e Organizações Internacionais de la PUC-RS.
}

\footnotetext{
${ }^{\text {II }}$ Ferro, et al., 2006

III Clemente, 2004: 327

${ }^{\text {IV }}$ Fernández Luzuriaga, 2005

${ }^{\vee}$ Luján en Quiroga; França, 2010:141

${ }^{\mathrm{VI}}$ Lula da Silva, 2006

VII Luján en Quiroga; França, 2010:138

VIII Alvariza, 2012, 7 de Mayo

Ix Uruguay, 2013, 21 de Octubre

x Brasil, 2011, 30 de Mayo
}

Cadernos do Tempo Presente, n. 24, jun./jul. 2016, p. 110-119| http://www.seer.ufs.br/index.php/tempo 
LA “ERA PROGRESISTA” EN URUGUAY: ¿CAMBIOS O CONTINUIDADES EN LA POLÍTICA EXTERIOR HACIA BRASIL?

RAFAEL ALVARIZA ALLENDE

${ }^{\mathrm{XI}}$ Rousseff, 2011, 30 de Mayo

XII Uruguay, 2013, 21 de Octubre

XIII Uruguay, 2013, 10 de Mayo 